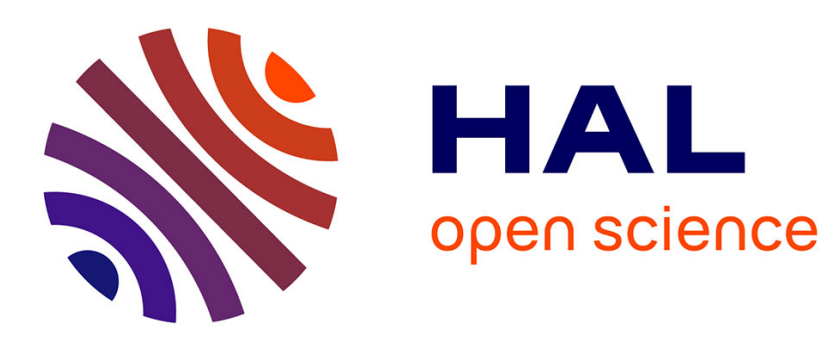

\title{
Burgers type equations, Gelfand's problem and Schumpeterian dynamics
}

Guennadi Khenkine

\section{To cite this version:}

Guennadi Khenkine. Burgers type equations, Gelfand's problem and Schumpeterian dynamics. 2012. hal-00692144

\section{HAL Id: hal-00692144 \\ https://hal.science/hal-00692144}

Preprint submitted on 28 Apr 2012

HAL is a multi-disciplinary open access archive for the deposit and dissemination of scientific research documents, whether they are published or not. The documents may come from teaching and research institutions in France or abroad, or from public or private research centers.
L'archive ouverte pluridisciplinaire HAL, est destinée au dépôt et à la diffusion de documents scientifiques de niveau recherche, publiés ou non, émanant des établissements d'enseignement et de recherche français ou étrangers, des laboratoires publics ou privés. 


\section{Burgers type equations, Gelfand's problem and Schumpeterian dynamics G.M.Henkin}

Université Pierre et Marie Curie, Paris, France

CEMI, Academy of Science, Moscow, Russia

Abstract. Burgers equations have been introduced to study different models of fluids Bateman, 1915, Burgers, 1939, Hopf, 1950, Cole, 1951, Lighthill, Whitham, 1955.... The difference-differential analogs of these equations have been proposed for Schumpeterian models of economic development Iwai, 1984, Polterovich, Henkin, 1988, Belenky, 1990, 1996, Henkin, Polterovich, 1999, Shananin, Tashlitskaya, 2000....

This paper is a survey of recent results and conjectures on Burgers type equations, motivated both by fluid mechanics and by Schumpeterian dynamics. Abriged proofs of new results are given. This paper is an extended version of the paper [H2] prepared for the talk at the conference "General Equilibrium Analysis" at Higher School of Economics, June, 2011.

1. Introduction: Burgers type equations and Schumpeterian dynamics.

By Burgers type equations we mean scalar partial differential equations of the form

$$
\frac{\partial f}{\partial t}+\varphi(f) \frac{\partial f}{\partial x}=\varepsilon \frac{\partial^{2} f}{\partial x^{2}}, \quad \varepsilon>0
$$

$f=f(x, t), x \in \mathbb{R}, t \in \mathbb{R}_{+}$, and the scalar difference-differential equations of the form

$$
\frac{\partial f}{\partial t}+\varphi(f) \frac{f(x, t)-f(x-\varepsilon, t)}{\varepsilon}=0, \quad x=k \varepsilon, k \in \mathbb{Z}
$$

and also variations and multidimensional generalization of (1a), (1b).

For linear $f \mapsto \varphi(f)$ equation (1a) was introduced by H.Bateman, 1915, and deeply studed by J.Burgers, 1939, as a simplest model for pressure-less gas dynamics. For general $\varphi(f)$ equation (1a) has appeared later in very different models, for example: in the model for displacement of oil by water (S.Buckley, M.Leverett, 1942), in the model of consolidation of wet soil (Florin, 1948), in the model of the road traffic (M.Lighthill, G.Whitham, 1955) etc.

The equation (1b) was proposed in Polterovich, Henkin, 1988,1989 for the description of a Schumpeterian evolution of industry.

According to Schumpeter 1911, 1939, economical development of industry is periodical process with period of order half-century ("business cycle"). It consists in cascades of creation, processes of formation and cascades of destruction. Creative and destructive cascades can be described by Lotka-Volterra type equations (P.Hanel, P.Klimek, S.Thurner, $2008,2010)$.

The mechanism of technological changes in the industry during processes of formation can be divided into two components: creation of new technologies by a firm (innovation process) and adoption of technologies, created by other firms (imitation process). For an 
industry with many firms its development can be described as evolution of its efficiency distribution. Let $f_{n}(t)$ be a share of firms at the moment $t$ in the given industry that have efficiency level $\leq n$.

The simplest model of Schumpeterian dynamics of industry has the form

$$
\frac{d f_{n}}{d t}=\varphi\left(f_{n}\right)\left(f_{n-1}(t)-f_{n}(t)\right), \text { where } \varphi\left(f_{n}\right)=\alpha+\beta\left(1-f_{n}\right),
$$

$\alpha \geq 0$ be the share of firms moving from level $n$ to the level $(n+1)$ per unit of time due to innovation, $\beta\left(1-f_{n}\right)$ be the share of firms moving from level $n$ to the level $(n+1)$ per unit of time due to imitation.

In applications this model may appear in different forms.

In the model with depreciation of capacities (Polterovich, Henkin, 1988) the following modification of the simplest model has appeared:

$$
\frac{d f_{n}}{d t}=\varphi\left(f_{n}\right)\left(f_{n-1}(t)-f_{n}(t)\right)+\mu\left(f_{n+1}(t)-f_{n}(t)\right)
$$

where $\mu$ is depreciation rate.

This model was succesfully implemented numerically (Gelman, Levin, Polterovich, Spivak, 1993) for description of evolution of distribution by efficiency levels for the Ferrous Metallurgy in USSR. Using automodel reduction $f_{n}(t)=f(t, n h)$ with $h \rightarrow 0$, A.Gasnikov and A.Shananin (2006) have found hypothetique formula for the velocity of travelling wave propagation for $(*)$.

In the model of economics growth (Polterovich, Henkin, 1989) we put $f_{n}=\sum_{k=0}^{n} M_{k} / \sum_{k=0}^{\infty} M_{k}$, where $M_{n}$ are capacities of the level $n$, and we have

$$
\frac{d M_{n}(t)}{d t}=\left(1-\varphi_{0}\left(f_{n}\right)\right) \lambda_{n} M_{n}+\varphi_{0}\left(f_{n-1}\right) \lambda_{n-1} M_{n-1}
$$

Here $\lambda_{n}$ - profit per unit of capacities per unit of time, $\varphi_{0}\left(f_{n}\right)$ - the share of profit $\lambda_{n} M_{n}$, creating new capacities of the level $n+1$. model

If $\lambda_{k} \uparrow \lambda>0$ and $\sum_{k=1}^{\infty} k\left(\lambda-\lambda_{k}\right)<\infty$, this gives the following variation of the simplest

$$
\frac{d f_{n}}{d t}=\varphi\left(f_{n}\right)\left(f_{n-1}-f_{n}\right)+r_{n},
$$

where $\varphi=\lambda \varphi_{0}$ and $r_{n}$ is a term nonessential for asymptotic behaviour.

In the model of Belenky, 1996, the speed of transition from efficiency level $n$ to level $(n+1)$ depends on a proportion $r_{n}=\left(1-f_{n}\right)\left(1-f_{n-1}\right)^{-1}$ of more advanced firms among all firms that are not worse than the firms of level $n$. This gives an interesting alternative for equation $(1 b)$ :

$$
\frac{d}{d t} \ln r_{n}(t)=\psi\left(r_{n}\right)-\psi\left(r_{n-1}\right)
$$

where $\psi$ is "motivation function", similar to function $\varphi$ in (1b). 
For physical applications of equation (1a) the main interest has the inviscid case of (1a), when $\varepsilon=+0$. But for transport flow models and for some social and biological applications the significant interest has the equation (1b) with $\varepsilon=1$ and $x \in \mathbb{Z}$.

The results of finite-difference approximations for nonlinear conservation laws (see A.Harten, J.Hyman, P.Lax, 1976, Engquist, Osher, 1981, Henkin, Shananin, 2004) explain both the similarity of behavior of (1a), (1b) and also some difference in behavior of (1a) and (1b).

Motivated by models of fluid mechanics, Gel'fand (1959) has formulated following problem.

To find asymptotic $(t \rightarrow \infty)$ of the solution $f(x, t)$ of the equation (1a) with initial condition

$$
f(x, 0)=\left\{\begin{array}{cl}
\alpha^{ \pm}, & \text {if } \pm x> \pm x^{ \pm} \\
f^{0}(x), & \text { if } x \in\left[x^{-}, x^{+}\right]
\end{array}\right.
$$

where $\alpha^{-} \leq \alpha^{+}, x^{-} \leq x^{+}$and $f^{0}$ is a bounded function on $\left[x^{-}, x^{+}\right]$.

Gelfand (1959) has found a solution of this problem for the inviscid case $\varepsilon=+0$ with special initial conditions $f(x, 0)=\alpha^{ \pm}$, if $\pm x>0$, and has noted:

it would be interesting to prove that the main term of asymptotic $(t \rightarrow \infty)$ of $f(x, t)$ satisfying (1a), (2) coincides with the solution of (1a), (2) with $\varepsilon=+0$.

Motivated by models of economical development similar problems were considered later for equation (1b) in Henkin, Polterovich (1991, 1994, 1999). For formulations of precise conjectures and results, concerning equations (1a) and (1b) we will use assumptions and definitions going back to Gelfand.

\section{Gelfand's problem. Main results.}

In the asymptotics statements below we will not indicate as a rule the dependence of some constants from initial function $f^{0}(x)$ and sometimes from state function $\varphi(f)$.

\section{Assumption 1.}

Let $\alpha^{-}<\alpha^{+}$and $\varphi$ be a positive continuous differentiable function on the interval $\left[\alpha^{-}, \alpha^{+}\right]$and $\varphi^{\prime}$ has only isolated zeros.

Put

$$
\begin{aligned}
& \psi(u)=-\int_{\alpha^{-}}^{u} \varphi(y) d y, u \in\left[\alpha^{-}, \alpha^{+}\right], \text {for }(1 . a), \\
& \psi(u)=\int_{\alpha^{-}}^{u} \frac{d y}{\varphi(y)}, \quad u \in\left[\alpha^{-}, \alpha^{+}\right], \text {for }(1 . b) .
\end{aligned}
$$

Let us introduce respectively for (3a) and for (3b) the concave function $\hat{\psi}(u)$ as the upper bound of the convex hull of the set

$$
\left\{(u, v): v \leq \psi(u), \quad u \in\left[\alpha^{-}, \alpha^{+}\right]\right\}
$$


Assumption 2. For (3a) and (3b) the set $S=\left\{u \in\left[\alpha^{-}, \alpha^{+}\right]: \psi(u)<\hat{\psi}(u)\right\}$ has the following form

$$
\begin{aligned}
& S=\left(\alpha_{0}^{-}, \alpha_{0}^{+}\right) \cup\left(\alpha_{1}^{-}, \alpha_{1}^{+}\right) \cup \ldots\left(\alpha_{L}^{-}, \alpha_{L}^{+}\right), \text {where } \\
& \alpha^{-}=\alpha_{0}^{-} \leq \alpha_{0}^{+}<\alpha_{1}^{-}<\alpha_{1}^{+}<\ldots<\alpha_{L-1}^{-}<\alpha_{L-1}^{+}<\alpha_{L}^{-} \leq \alpha_{L}^{+}=\alpha^{+} .
\end{aligned}
$$

Let

$$
\begin{aligned}
& c_{l}=\frac{1}{\alpha_{l}^{+}-\alpha_{l}^{-}} \int_{\alpha_{l}^{-}}^{\alpha_{l}^{+}} \varphi(y) d y, \text { for }(1 a), \quad l=0, \ldots, L, \\
& c_{l}=\left(\alpha_{l}^{+}-\alpha_{l}^{-}\right)\left(\int_{\alpha_{l}^{-}}^{\alpha_{l}^{+}} \frac{d y}{\varphi(y)}\right)^{-1}, \text { for }(1 b), l=0, \ldots, L .
\end{aligned}
$$

Assumptions 1,2 imply (Gelfand, 1959, Oleinik, 1959, Weinberger, 1990, Henkin, Polterovich, 1999) the following important inequalities (for (1a)) and respectively for (1b):

$$
\begin{aligned}
& \varphi\left(\alpha_{l}^{+}\right) \leq c_{l} \leq \varphi\left(\alpha_{l}^{-}\right), \quad l=0, \ldots, L \\
& c_{l}=\varphi\left(\alpha_{l}^{-}\right), \quad l=1, \ldots, L \\
& c_{l}=\varphi\left(\alpha_{l}^{+}\right), \quad l=0, \ldots, L-1 .
\end{aligned}
$$

Let us remark that the inequalities above are, in fact, equalities except for the cases $l=0$ and $l=L$.

Assumption 3. Let for (1a) and respectively for (1b) the following inequalities are valid

$$
\begin{aligned}
& \varphi^{\prime}\left(\alpha_{l}^{-}\right) \neq 0, \quad l=1, \ldots, L, \\
& \varphi^{\prime}\left(\alpha_{l}^{+}\right) \neq 0, \quad l=0,1, \ldots, L-1, \\
& \varphi\left(\alpha_{0}^{-}\right) \neq c_{0}, \quad \text { if } \alpha_{0}^{-}<\alpha_{0}^{+}, \\
& \varphi\left(\alpha_{L}^{+}\right) \neq c_{L}, \quad \text { if } \alpha_{L}^{-}<\alpha_{L}^{+} .
\end{aligned}
$$

Theorem 1. (Generalized Gelfand theorem).

Let under assumptions 1-3 we have $\varepsilon=+0$. Let, in addition, initial data function $f^{0}(x)$ be the function of bounded variation on $\left[x^{-}, x^{+}\right]$. Then solutions of the Cauchy problems $(1 \mathrm{a}, \mathrm{b}),(2)$ have the following asymptotic structure

$$
f(x, t) \stackrel{L^{1}(\mathbb{R})}{\Rightarrow}\left\{\begin{array}{cl}
\alpha^{-}, & \text {if } x<c_{0} t+d_{0} \\
\varphi^{(-1)}(x / t), & \text { if } c_{l} t+d_{l} \leq x<c_{l+1} t+d_{l+1}, l=0,1, \ldots, L-1 \\
\alpha^{+}, & \text {if } x \geq c_{L} t+d_{L},
\end{array}\right.
$$

where $t \rightarrow \infty$, parameters $\left\{c_{l}\right\}$ determined by $(5 \mathrm{a}, \mathrm{b})$ and parameters $\left\{d_{l}\right\}$ determined by corresponding equation $(1 \mathrm{a}, \mathrm{b})$ and initial data $(2)$, inverse function $\varphi^{(-1)}(\cdot)$ is well defined on $\left[c_{l}, c_{l+1}\right], 0 \leq l \leq L-1$. 
- For equation (1a) with special initial condition $f(x, 0)=\alpha^{ \pm}$, if $\pm x>0$

Theorem 1 was obtained by Gelfand, 1959. Theorem 1 under condition $\alpha^{-}=\alpha_{0}^{-}<\alpha_{0}^{+}=$ $\alpha^{+}$was proved by Iljin, Oleinik, 1960, for equation (1a) and can be deduced from Henkin, Polterovich, 1991 for equation (1b).

- Under additional assumption that $f(x, t)$ is piecewise smooth function of $(x, t) \in$ $\mathbb{R} \times \mathbb{R}^{+}$and $f^{0}(x) \in\left[\alpha^{-}, \alpha^{+}\right], x \in\left[x^{-}, x^{+}\right]$, Theorem 1 was obtained by Kruzhkov, Petrosyan, 1983, 87. 2009 .

- Theorem 1 for equation (1a) only under assumption 1 was announced by Gasnikov,

- Theorem 1 for both equations (1a) and (1b) is deduced here by vanishing viscocity method from results of [H1] (see Theorems 2, 3 below). It gives the answer to the question formulated in [KP] ("...the ways of application of vanishing viscocity methods in this case are absolutely unknown...").

For formulation of main results (Theorems 2,3) we need the following important statement about travelling waves solutions of equations (1a,b) belonging to Gelfand, 1959, Oleinik, 1959, for equation (1a) and Polterovich, Henkin, 1988, Belenky, 1990, for equation (1b).

\section{Proposition 1.}

Under assumptions 1,2 for $l \in\{0, \ldots, L\}$ there exist (and unique up to translations) travelling wave solutions of (1a) and (1b) of the form

$$
\begin{aligned}
& f=\tilde{f}_{l}\left(\frac{x-c_{l} t}{\varepsilon}\right) \text { such that } \forall \varepsilon>0 \\
& \tilde{f}_{l}\left(\frac{x}{\varepsilon}\right) \rightarrow \alpha_{l}^{ \pm} \text {as } x \rightarrow \pm \infty, l=0, \ldots, L, \text { and } \\
& \tilde{f}_{l}\left(\frac{x}{\varepsilon}\right) \rightarrow \alpha_{l}^{ \pm} \text {as } \varepsilon \rightarrow 0, \text { if } \pm x>0 .
\end{aligned}
$$

\section{Theorem 2.}

Let $\varepsilon \in(0,1)$. Under the assumptions $1,2,3$ and definitions $(3 \mathrm{a}, \mathrm{b}),(4 \mathrm{a}, \mathrm{b}),(5 \mathrm{a}, \mathrm{b})$ the solutions $f(x, t)$ of the Cauchy problems $(1 \mathrm{a}, \mathrm{b}),(2)$ have for $t \geq \varepsilon O\left(\left|\ln \frac{1}{\varepsilon}\right|^{2}\right)$ the following asymptotic structure:

$$
\begin{aligned}
& f(x, t)=O\left(\frac{\varepsilon}{t}\right)^{1 / 4}+ \\
& \left\{\begin{array}{cl}
\tilde{f}_{l}\left(\frac{1}{\varepsilon}\left(x-c_{l} t-d_{l}\left(\frac{t}{\varepsilon},\left(\frac{\varepsilon}{t}\right)^{1 / 4}\right)\right)\right), & \text { if }\left|x-c_{l} t\right| \leq \sqrt{\varepsilon} \cdot(\varepsilon t)^{1 / 4}, \quad l=0,1, \ldots, L, \\
\varphi^{(-1)}\left(\frac{x}{t}\right), & \text { if } c_{l} t+\sqrt{\varepsilon}(\varepsilon t)^{1 / 4} \leq x \leq c_{l+1} t-\sqrt{\varepsilon}(\varepsilon t)^{1 / 4}, \\
\alpha^{-}, & l=0,1, \ldots, L-1, \\
\alpha^{+}, & \text {if } x \leq c_{0} t-\sqrt{\varepsilon}(\varepsilon t)^{1 / 4,} \\
& \text { if } x \geq c_{L} t+\sqrt{\varepsilon}(\varepsilon t)^{1 / 4},
\end{array}\right.
\end{aligned}
$$

where shift functions $d_{l}\left(\frac{t}{\varepsilon}, A\right), A=\left(\frac{\varepsilon}{t}\right)^{1 / 4}$ are determined by the "localized conservation 
laws" (see [HS])

$$
\int_{c_{l} t-A \sqrt{\varepsilon t}}^{c_{l} t+A \sqrt{\varepsilon t}}\left[f(x, t)-\tilde{f}_{l}\left(\frac{1}{\varepsilon}\left(x-c_{l} t-d_{l}\left(\frac{t}{\varepsilon}, A\right)\right)\right)\right] d x=0
$$

for equation (1a) and

$$
\begin{aligned}
& \sum_{k=\left[c_{l} t-A \sqrt{\varepsilon t}\right]+1}^{\left[c_{l} t+A \sqrt{\varepsilon t}\right]-1}\left(\Phi(f(k \varepsilon, t))-\Phi\left(\tilde{f}_{l}\left(\frac{1}{\varepsilon}\left(k \varepsilon-c_{l} t-d_{l}\left(\frac{t}{\varepsilon}, A\right)\right)\right)\right) \pm\right. \\
& \left(c_{l} t \pm A \sqrt{\varepsilon t}-\left[c_{l} t \pm A \sqrt{\varepsilon t}\right]\right) \times \\
& \left(\Phi\left(f\left(\left[c_{l} t \pm A \sqrt{\varepsilon t}\right], t\right)\right)-\Phi\left(\tilde{f}_{l}\left(\left[c_{l} t \pm A \sqrt{\varepsilon t}\right]-c_{l} t-d_{l}\left(\frac{t}{\varepsilon}, A\right)\right)\right)\right)
\end{aligned}
$$

where $\Phi(f)=\int_{f}^{\alpha^{+}} \frac{d y}{\varphi(y)}$ for equation (1b).

\section{Theorem 3.}

Under conditions of Theorem 2 the shift functions $d_{l}\left(\frac{t}{\varepsilon},\left(\frac{\varepsilon}{t}\right)^{1 / 4}\right)$ have for $t \geq \varepsilon O\left(\ln \frac{1}{\varepsilon}\right)^{2}$ the following asymptotics:

$$
d_{l}\left(\frac{t}{\varepsilon},\left(\frac{\varepsilon}{t}\right)^{1 / 4}\right)=\varepsilon \gamma_{l} \ln \frac{t}{\varepsilon}+\varepsilon O\left(\ln \frac{t}{\varepsilon}\right)^{2 / 3}+O(1),
$$

where $\left\{\gamma_{l}\right\}$ are the parameters, depending on $\left\{\alpha_{l}^{ \pm}, \varphi\left(\alpha_{l}^{ \pm}\right), \varphi^{\prime}\left(\alpha_{l}^{ \pm}\right)\right\}$by explicit formulas for problem (1a), (2):

$$
\begin{gathered}
\gamma_{0}=\gamma_{0, a}=\left\{\begin{array}{cl}
0, & \text { if } L=0, \\
\frac{1}{\alpha_{0}^{+}-\alpha_{0}^{-}}\left(-\frac{2}{\varphi^{\prime}\left(\alpha_{0}^{+}\right)}\right), & \text {if } L>0 \text { and } \alpha_{0}^{-}<\alpha_{0}^{+},
\end{array}\right. \\
\gamma_{l}=\gamma_{l, a}=\frac{1}{\alpha_{l}^{+}-\alpha_{l}^{-}}\left(\frac{2}{\varphi^{\prime}\left(\alpha_{l}^{-}\right)}-\frac{2}{\varphi^{\prime}\left(\alpha_{l}^{+}\right)}\right), \quad l=1, \ldots, L-1, \\
\gamma_{L}=\gamma_{L, a}=\left\{\begin{array}{cl}
\frac{1}{\alpha_{L}^{+}-\alpha_{L}^{-}}\left(\frac{2}{\varphi^{\prime}\left(\alpha_{L}^{-}\right)}\right), & \text {if } L>0 \text { and } \alpha_{L}^{-}<\alpha_{L}^{+}, \\
0, & \text { if } L=0 .
\end{array}\right.
\end{gathered}
$$

For the problem (1b), (2) we have

$$
\gamma_{l}=\gamma_{l, b}=\frac{c_{l}}{2} \gamma_{l, a}, \quad l=0, \ldots, L
$$

\section{Comments}

- Theorems 2, 3 are improved versions of Theorems 1a,b from Henkin, 2007. 
- Theorems 2, 3 generalize the results of Iljin, Oleinik, 1960, Weinberger, 1990, Henkin, Polterovich, 1991, 1999, Henkin, Shananin, Tumanov, 2004, 2005, Engelberg, Schochet, 2006.

- Theorems 2, 3 answer to the important questions about location in the Cauchy problems (1a,b), (2) of viscous shock-waves Gelfand, 1959, Henkin, Polterovich, 1994, 1999, Liu, Matsumura, Nishihara, 1998.

- Theorems 2, 3 imply the new interesting phenomens: if $L>0$ and

$x \in\left[c_{l} t-A \sqrt{t}, c_{l} t+A \sqrt{t}\right], l \in\{0, \ldots, L\}$, then solutions of (1a,b), (2) converge to shifted travelling waves $\tilde{f}_{l}\left(\frac{1}{\varepsilon}\left(x-c_{l} t-\varepsilon \gamma_{l} \ln t-\varepsilon O(\ln t)^{2 / 3}+O(1)\right)\right)$, which generally do not satisfy equations $(1 \mathrm{a}, \mathrm{b})$ and the positions of which on the x-line depend essentially on the (viscosity) parameter $\varepsilon>0$. These phenomens lead to the appropriate correction of the Gelfand's suggestion that the main term of asymptotic $(t \rightarrow \infty)$ of $f(x, t)$, satisfying $(1 \mathrm{a}, \mathrm{b})$, coincides with solutions of $(1 \mathrm{a}, \mathrm{b})$ for $\varepsilon=+0$ with the same initial conditions.

- Basing on the works (Kolmogorov, Petrovski, Piskunov, 1937, and Mejai, Volpert, 1999), Gasnikov, 2008, 2009, has proved (only under assumption 1) a rough version of Theorem 2 with shift function $d_{l}(t)=o(t)$ instead of the precise shift function $d_{l}(t)=\varepsilon \gamma_{l} \ln t+\varepsilon O(\ln t)^{2 / 3}+O(1)$.

The proofs of Theorems 2, 3 are variations of the proofs of Theorems 1 a,b from [H1] and combine earlier techniques (maximum and comparison principles, Lyapunov type functions, Poisson-Green kernels for parabolic type equations) together with new ingredients. For the proof of Theorems 2,3 we need, first, the following comparison proposition from [H1], which is an improvement of Theorem 7.5 from [HP4].

\section{Proposition 1.}

Under assumption of Theorem 2 and assumption $\varepsilon=1$, solutions $f(x, t)$ of $(1 \mathrm{a}, \mathrm{b}),(2)$ satisfy the following estimates:

for every $\gamma>0$ and $b_{l}>O\left(\frac{1}{\gamma}\right), l=0, \ldots, L-1$, there exists $t_{0}=O\left(b_{l} \gamma\right)$ such that

$$
\varphi^{(-1)}\left(\frac{x-\gamma \sqrt{t}}{t}\right) \leq f(x, t) \leq \varphi^{(-1)}\left(\frac{x+\gamma \sqrt{t}}{t}\right)
$$

for $x \in\left[c_{l} t+b_{l} \sqrt{t}, c_{l+1} t-b_{l+1} \sqrt{t}\right]$ and $t \geq t_{0}$, where constants $c_{l}$ are defined by $(5 \mathrm{a}, \mathrm{b})$.

We need next a proposition, which improves the results of [HST] and of [H1], concerning a priori estimates of derivatives of solutions of the equations $(1 \mathrm{a}, \mathrm{b})$.

\section{Proposition 2.}

Under assumptions of Theorem 2 and of Proposition 1, let $\varepsilon=1, L>0, \varphi\left(\alpha_{l}^{+}\right)=c_{l}$, $l=0, \ldots, L-1, \varphi\left(\alpha_{l}^{-}\right)=c_{l}, l=1, \ldots, L$. Let $\tilde{b}_{l}>b_{l}>O\left(\frac{1}{\gamma}\right), l=0, \ldots, L, \gamma>0$.

Then the difference $\Delta f=f(x, t)-f(x-1, t)$ for solution of $(1 \mathrm{~b}),(2)$ and the derivative $\frac{\partial f}{\partial x}(x, t)$ for solution of (1a), (2) satisfy the following estimates:

$$
\left\{\begin{array}{c}
\Delta f \\
\frac{\partial f}{\partial x}
\end{array}\right\}=\frac{1}{\varphi^{\prime}\left(\alpha_{l}^{+}\right)(t+1)}+O\left(\frac{\gamma}{\varphi^{\prime}\left(\alpha_{l}^{+}\right)(t+1)}\right),
$$

for $x \in\left[c_{l} t+b_{l} \sqrt{t}, c_{l} t+\tilde{b}_{l} \sqrt{t}\right], l=0, \ldots, L-1, t \geq t_{0}$, and

$$
\left\{\begin{array}{c}
\Delta f \\
\frac{\partial f}{\partial x}
\end{array}\right\}=\frac{1}{\varphi^{\prime}\left(\alpha_{l}^{-}\right)(t+1)}+O\left(\frac{\gamma}{\varphi^{\prime}\left(\alpha_{l}^{-}\right)(t+1)}\right),
$$


for $x \in\left[c_{l} t-\tilde{b}_{l} \sqrt{t}, c_{l} t-b_{l} \sqrt{t}\right], l=1, \ldots, L, t \geq t_{0}$, where $t_{0}=O\left(\frac{\tilde{b}_{l}}{b_{l}}+b_{l} \gamma\right)$.

The proof of Proposition 2 for solutions of (1b), (2), given in Section 4, develops and corrects the proofs of the corresponding statements in [HST] and [H1].

Propositions 1, 2 imply the following improved version of lemmas 11, 12 from [H1].

\section{Proposition 3.}

Under assumptions of Theorems 1,2 for any $A>0, \theta \in[0,1)$ and $l \in\{0, \ldots, L\}$ the following estimates for shift functions $d_{l}(t, A)$, defined by $(8 \mathrm{a}, \mathrm{b})$, are valid

$$
\begin{aligned}
& \left|\frac{\partial}{\partial t} d_{l}(t, A)\right|=\frac{\gamma_{l, a}}{(t+1)}+O\left(\frac{1}{A(t+1)}\right), \\
& \left|\int_{t-\theta}^{t+1-\theta} \frac{\partial}{\partial \tau} d_{l}(\tau, A) d \tau\right|=\frac{\gamma_{l, b}}{(t+1)}+O\left(\frac{1}{A(t+1)}\right), \\
& \left|\frac{\partial}{\partial A} d_{l}(t, A)\right|=O\left(A+\frac{1}{A}\right), \quad t \geq t_{0},
\end{aligned}
$$

where $\gamma_{l, a}, \gamma_{l, b}$ the parameters, defined in Theorem 3.

The following Proposition 4, precised version of Proposition 6 from [H1], gives the main element of the proofs of Theorems 2, 3 .

\section{Proposition 4.}

Let $f$ be solution of the Cauchy problem (1a), (2) or (1b), (2), where $\varepsilon>0$. Then under assumptions of Theorem 2 and Proposition 1 we have convergence of $f(x, t)$ to the shifted travelling waves $\tilde{f}_{l}\left(\frac{1}{\varepsilon}\left(x-c_{l} t-d_{l}\left(\frac{t}{\varepsilon}, \sqrt{\delta}\right)\right)\right), l=0, \ldots, L$, on the intervals $\left\{x \in \mathbb{R}:\left|x-c_{l} t\right| \leq \sqrt{\delta t \varepsilon}\right\}$ with the estimates

$$
\sup _{\left\{x:\left|x-c_{l} t\right| \leq \sqrt{\delta t \varepsilon}\right\}}\left|f_{l}(x, t)-\tilde{f}_{l}\left(\frac{1}{\varepsilon}\left(x-c_{l} t-d_{l}\left(\frac{t}{\varepsilon}, \sqrt{\delta}\right)\right)\right)\right|=O(\sqrt{\delta}), \quad \text { if } \quad t \geq t_{0}=\varepsilon O\left(\ln \frac{1}{\delta}\right)^{2} .
$$

Idea of the proof of Proposition 4 for solution $f(k, t)$ of equation (1b), (2) with $\varepsilon=1$ and $k \in \mathbb{Z}$ consists (see [H1]) in proving of the following estimate : $\forall \delta>0$ and $\forall l \in$ $\{0,1, \ldots, L\}$

$$
\varlimsup_{t \rightarrow \infty} \sup _{\left\{n:\left|n-c_{l} t\right| \leq \sqrt{\delta t}\right\}} \mid \sum_{\left[c_{l} t-\sqrt{\delta t}\right]}^{n}\left(\Phi(f(k, t))-\Phi\left(\tilde{f}_{l}\left(k-c_{l} t-d_{l}(t, \sqrt{\delta})\right)\right) \mid \leq O(\sqrt{\delta}) .\right.
$$

The proof of (12) uses nonlinear parabolic type equations for the functions

$$
\Delta_{l}\left(n, t, d_{l}(\tau, \sqrt{\delta})\right)=\sum_{k=\left[c_{l} \tau-\sqrt{\delta \tau}\right]}^{n}\left(\Phi(f(k, t))-\Phi\left(\tilde{f}_{l}\left(n-c_{l} t-d_{l}(\tau, \sqrt{\delta})\right)\right)\right.
$$

of variables $n, t$ in the domain $n \in\left[c_{l} \tau-\sqrt{\delta \tau}, c_{l} t+\sqrt{\delta t}\right] \cap \mathbb{Z}, t \in(\tau, \tau+\sqrt{\delta \tau})$. Localized conservation laws are used in order to have a priori boundary estimates:

$$
\left|\Delta_{l}\left(\left[c_{l} t+\sqrt{\delta t}\right], t, d_{l}(\tau, \sqrt{\delta})\right)\right|=O(1 / \sqrt{\tau}) .
$$


The estimate (12) implies uniform convergence $f(n, t) \Rightarrow \tilde{f}_{l}\left(n-c_{l} t-d_{l}(t, o(1))\right)$ in intervals $\left|n-c_{l} t\right| \leq o(\sqrt{t}), l=0, \ldots, L$.

\section{Proofs of Theorems 1, 2, 3 .}

\section{Proof of Theorem 3.}

Let $0<A_{0}<1<A, \varepsilon=1, l=0, \ldots, L$. Estimates (10a,b) from Proposition 3 imply the following

$$
d_{l}(t, A)=\gamma_{l} \ln \frac{t}{t_{0}}+O\left(\frac{1}{A} \ln \frac{t}{t_{0}}\right)+d_{l}\left(t_{0}, A\right) .
$$

Estimate (11) from Proposition 3 implies

$$
d_{l}\left(t, A_{0}\right)=d_{l}(t, A)-O_{t}\left(A^{2}\right)-O_{t}\left(\ln \frac{1}{A_{0}}\right), \text { where } t \geq t_{0}
$$

From estimates (13), (14) we deduce

$$
d_{l}\left(t, A_{0}\right)=d_{l}\left(t_{0}, A\right)+\gamma_{l} \ln \frac{t}{t_{0}}+O\left(\frac{1}{A} \ln \frac{t}{t_{0}}\right)-O_{t}\left(A^{2}\right)-O_{t}\left(\ln \frac{1}{A_{0}}\right) .
$$

Putting in this estimate $A=\left(\ln \frac{t}{t_{0}}\right)^{1 / 3}$ we obtain

$$
d_{l}\left(t, A_{0}\right)=\gamma_{l} \ln \frac{t}{t_{0}}+O_{t}\left(\left(\ln \frac{t}{t_{0}}\right)^{2 / 3}\right)+d_{l}\left(t_{0}, A\right)-O_{t}\left(\ln \frac{1}{A_{0}}\right)
$$

Putting in (15) $t=t_{0}$ we obtain

$$
d_{l}\left(t_{0}, A_{0}\right)=d_{l}\left(t_{0}, A\right)-O_{t_{0}}\left(\ln \frac{1}{A_{0}}\right)
$$

Let us make the rescalling of equation (15) with parameters $\varepsilon=1, \tilde{t}, \tilde{x}, \tilde{d}, \tilde{A}, \tilde{t}_{0}$ into equation with parameters $\varepsilon>0, t, x, d, A, t_{0}$, using relations

$$
\tilde{t}=\frac{t}{\varepsilon}, \tilde{x}=\frac{x}{\varepsilon}, \tilde{d}=\frac{d}{\varepsilon}, \tilde{A}=\frac{A}{\sqrt{\varepsilon}}, \tilde{t}_{0}=\frac{t_{0}}{\varepsilon}=1
$$

We obtain

$$
d_{l}\left(\frac{t}{\varepsilon}, A_{0}\right)=d_{l}\left(1, A_{0}\right)+\varepsilon \gamma \ln \frac{t}{\varepsilon}+\varepsilon\left(\ln \frac{t}{\varepsilon}\right)^{2 / 3}-O\left(\ln \frac{1}{A_{0}}\right) .
$$

From Proposition 4 we deduce the following estimate

$$
\begin{aligned}
& \sup _{\left\{x:\left|x-c_{l} t\right| \leq \sqrt{\varepsilon}(\varepsilon t)^{1 / 4}\right\}}\left|\tilde{f}_{l}\left(\frac{1}{\varepsilon}\left(x-c_{l} t-d_{l}\left(\frac{t}{\varepsilon},\left(\frac{\varepsilon}{t}\right)^{1 / 4}\right)\right)\right)-\tilde{f}_{l}\left(\frac{1}{\varepsilon}\left(x-c_{l} t-d_{l}\left(\frac{t}{\varepsilon}, A_{0}\right)\right)\right)\right|= \\
& O\left(A_{0}\right), \text { if } t \geq t_{0} \geq \varepsilon O\left(\ln \frac{1}{A_{0}}\right)^{2} .
\end{aligned}
$$


Last estimate implies

$$
d_{l}\left(\frac{t}{\varepsilon}, A_{0}\right)=d_{l}\left(\frac{t}{\varepsilon},\left(\frac{\varepsilon}{t}\right)^{1 / 4}\right)+O\left(A_{0}\right), \text { if } t \geq \varepsilon O\left(\ln \frac{1}{A_{0}}\right)^{2} .
$$

From estimates (16), (17) we obtain

$$
\begin{aligned}
& d_{l}\left(\frac{t}{\varepsilon},\left(\frac{\varepsilon}{t}\right)^{1 / 4}\right)=d_{l}\left(1, A_{0}\right)+\varepsilon \gamma \ln \frac{t}{\varepsilon}+\varepsilon\left(\ln \frac{t}{\varepsilon}\right)^{2 / 3}+O\left(A_{0}\right)+O\left(\ln \frac{1}{A_{0}}\right), \\
& \text { if } t \geq t_{0} \geq \varepsilon O\left(\ln \frac{1}{A_{0}}\right)^{2} .
\end{aligned}
$$

This gives statement of Theorem 3 with

$$
O(1)=d_{l}\left(1, A_{0}\right)+0\left(\ln \frac{1}{A_{0}}\right)
$$

\section{Proof of Theorem 2.}

Let $\varepsilon=1$. Let $f$ be solution of $(1 \mathrm{a}),(2)$ or $(1 \mathrm{~b}),(2)$ and $\left\{\tilde{f}_{l}\right\}$ be travelling waves solutions for (1a) or (1b) from Proposition 1. Then Proposition 6 from [H1] (i.e. Proposition 4 above with $\varepsilon=1$ ) implies $\forall \delta>0$ estimate

$$
\sup _{l} \sup _{\left\{x:\left|x-c_{l} t\right| \leq \sqrt{\delta t}\right\}}\left|f(x, t)-\tilde{f}_{l}\left(x-c_{l} t-d_{l}(t, \sqrt{\delta})\right)\right|=O(\sqrt{\delta}), \quad \text { if } \quad t \geq t_{0}(\delta) .
$$

Crucial inequality (6.12) in [H1] shows that one can take in (19)

$$
t_{0}(\delta)=0\left(\ln \frac{1}{\delta}\right)^{2}
$$

Results of [W] and [HP4] imply estimates

$$
\begin{aligned}
& \left|f(x, t)-\varphi^{(-1)}\left(\frac{x}{t}\right)\right|=O\left(\frac{1}{\sqrt{\delta t}}\right), \quad \text { if } \quad c_{l} t+\sqrt{\delta t} \leq x \leq c_{l+1} t-\sqrt{\delta t}, \quad l=0, \ldots, L-1, \\
& \text { and }\left|f(x, t)-\alpha_{ \pm}\right|=O\left(\frac{1}{\sqrt{\delta t}}\right), \quad \text { if } \quad x \leq c_{l} t-\sqrt{\delta t} \text { or } x \geq c_{L} t+\sqrt{\delta t} .
\end{aligned}
$$

Making the rescalling $\tilde{t}=\frac{t}{\varepsilon}, \tilde{x}=\frac{x}{\varepsilon}, \tilde{d}=\frac{d}{\varepsilon}$, and $\delta=\sqrt{\frac{\varepsilon}{t}}$ into (19), (20), (21) we obtain statement of Theorem 2 , if

$$
\frac{t_{0}}{\varepsilon} \geq\left(\ln t_{0}+\ln \frac{1}{\varepsilon}\right)^{2}, \text { i.e. if } t_{0} \geq \text { constant }
$$

\section{Proof of Theorem 1.}


Let $f^{0}(x, t)$ be solution of the Cauchy problem (1a), (2) with $\varepsilon=+0$. Let $\delta>0$, $\varepsilon_{k}=\delta e^{-k}$ and $t_{k}=k, k=0,1,2, .$. Let us define continuous function $f(x, t)$ for $x \in \mathbb{R}$, $t \in \mathbb{R}^{+}$by the following procedure. For $t \in\left[t_{k}, t_{k+1}\right]$ we put $f(x, t)=f_{k}(x, t)$ such that

$$
\begin{aligned}
& f_{0}(x, 0)=f^{0}(x, 0)=f^{0}(x) \text { and } \\
& \frac{\partial f_{k}}{\partial t}+\varphi\left(f_{k}\right) \frac{\partial f_{k}}{\partial x}=\varepsilon_{k} \frac{\partial^{2} f_{k}}{\partial x^{2}}, \quad \text { if } t \in\left[t_{k-1}, t_{k}\right], \quad k+1,2, . .
\end{aligned}
$$

Applying inductively (22) and result of Kuznetsov, 1975, we obtain for $t \leq t_{k}$ the estimate

$$
\int_{x \in \mathbb{R}}\left|f(x, t)-f^{0}(x, t)\right| \leq O\left(T V f^{0}\right)\left(\sqrt{\varepsilon_{1} t_{1}}+\sqrt{\varepsilon_{2} t_{2}}+\ldots+\sqrt{\varepsilon_{k} t_{k}}\right),
$$

where $T V f^{0}$ means a total variation of $f^{0}(x)$ on $\mathbb{R}$. Substitution of $\varepsilon_{k}=\delta e^{-k}$ and $t_{k}=k$ in (23) imply estimate

$$
\int_{x \in \mathbb{R}}\left|f(x, t)-f^{0}(x, t)\right|=O(\sqrt{\delta}) T V\left(f^{0}\right), \quad t \in \mathbb{R}^{+} .
$$

Using equations (22) and Theorem 2 we obtain for $t \in\left[t_{k-1}, t_{k}\right]$ and $x \in\left[c_{l} t-\sqrt{\varepsilon_{k}}\left(\varepsilon_{k} t\right)^{1 / 4}, c_{l} t+\sqrt{\varepsilon_{k}}\left(\varepsilon_{k} t\right)^{1 / 4}\right]$ inequalities

$$
\left|f_{k}(x, t)-\tilde{f}_{k, l}\left(\frac{1}{\varepsilon_{k}}\left(x-c_{l} t-d_{l}\left(\frac{t}{\varepsilon_{k}},\left(\frac{\varepsilon_{k}}{t}\right)^{1 / 4}\right)\right)\right)\right|=O\left(\frac{\varepsilon_{k}}{t}\right)^{1 / 4}, \quad k=1,2, \ldots, \quad l=0,1, \ldots, L .
$$

If $x \in\left[c_{l} t+\sqrt{\varepsilon_{k}}\left(\varepsilon_{k} t\right)^{1 / 4}, c_{l} t-\sqrt{\varepsilon_{k}}\left(\varepsilon_{k} t\right)^{1 / 4}\right]$ we obtain inequality

$$
\left|f(x, t)-\varphi^{(-1)}\left(\frac{x}{t}\right)\right|=O\left(\frac{\varepsilon_{k}}{t}\right)^{1 / 4}, \quad l=0,1, \ldots, L-1 .
$$

We have also inequalities

$$
\begin{aligned}
& \left|f(x, t)-\alpha^{-}\right|=O\left(\frac{\varepsilon_{k}}{t}\right)^{1 / 4} \text { and }\left|f(x, t)-\alpha^{+}\right|=O\left(\frac{\varepsilon_{k}}{t}\right)^{1 / 4}, \text { if } \\
& x \leq c_{l} t-\sqrt{\varepsilon_{k}}\left(\varepsilon_{k} t\right)^{1 / 4} \text { or } x \geq c_{l} t+\sqrt{\varepsilon_{k}}\left(\varepsilon_{k} t\right)^{1 / 4} .
\end{aligned}
$$

From Theorem 3 (more precisely from equality (18) implying Theorem 3) we have inequalities

$$
d_{l}\left(\frac{t}{\varepsilon},\left(\frac{\varepsilon_{k}}{t}\right)^{1 / 4}\right)=O\left(\varepsilon_{k} \ln \frac{t}{\varepsilon_{k}}\right)+O\left(A_{0}\right)+O\left(\ln \frac{1}{A_{0}}\right), \quad \text { if } t \geq t_{0} \geq \varepsilon O\left(\ln \frac{1}{A_{0}}\right)^{2} .
$$

From (24)-(27) we deduce that $\forall \delta>0, t \in[k-1, k], l=0,1, \ldots, L-1$, the following asymptotic equality holds

$$
\begin{aligned}
& f^{0}(x, t)=O(\sqrt{\delta})+O\left(\frac{\varepsilon_{k}}{t}\right)^{1 / 4}+\tilde{f}_{l}\left(\frac{1}{\varepsilon_{k}}\left(x-c_{l} t-O\left(\varepsilon_{k} \ln \frac{t}{\varepsilon_{k}}\right)-O\left(A_{0}\right)-O\left(\ln \frac{1}{A_{0}}\right)\right)\right), \quad \text { if } \\
& x \in\left[c_{l} t-\sqrt{\varepsilon_{k}}\left(\varepsilon_{k} t\right)^{1 / 4}, c_{l} t+\sqrt{\varepsilon_{k}}\left(\varepsilon_{k} t\right)^{1 / 4}\right], \\
& f^{0}(x, t)=\varphi^{(-1)}\left(\frac{x}{t}\right), \text { if } x \in\left[c_{l} t-\sqrt{\varepsilon_{k}}\left(\varepsilon_{k} t\right)^{1 / 4}, c_{l+1} t+\sqrt{\varepsilon_{k}}\left(\varepsilon_{k} t\right)^{1 / 4}\right] .
\end{aligned}
$$


From (28) we obtain the existence of constants $d_{l}^{*}$ such that

$$
f^{0}(x, t) \stackrel{L^{1}}{\stackrel{\mathbb{R}), t \rightarrow \infty}{\Longrightarrow}}\left\{\begin{array}{cl}
\alpha^{-}, & \text {if } x<c_{0} t+d_{0}^{*} \\
\varphi^{(-1)}(x / t), & \text { if } c_{l} t+d_{l}^{*} \leq x<c_{l+1} t+d_{l+1}^{*}, l=0,1, \ldots, L-1 \\
\alpha^{+}, & \text {if } x \geq c_{L} t+d_{L}^{*} .
\end{array}\right.
$$

\section{Remark.}

We do not use in our proof apriori condition that $f^{0}(x, t)$ is a piecewise smooth solution of (1), (2) with $\varepsilon=0$ like in $[\mathrm{P}],[\mathrm{KP}]$. But results of $[\mathrm{P}],[\mathrm{KP}]$ have advantage, giving explicit formulas for constants $\left\{d_{l}^{*}\right\}$.

\section{Proof of Proposition 2.}

We give here the proof of Proposition 2 precising and correcting arguments of [HST] and [H1]. The main tool is as in [HST], [H1] the Green-Poisson type formula associated with operator $u_{t}^{\prime}+\Delta u$.

Lemma 1 ([HS],p.1475).

Let $u(x, t)$ be a function defined in the domain $\Omega=\left\{(x, t): t>0, b<\bar{x} \stackrel{\text { def }}{=} \frac{x-t}{\sqrt{t}}<\tilde{b}+\sigma \sqrt{t}\right\}, \sigma>\sigma_{0}>0$. Let $\chi(x, t)=\chi_{0}\left(\frac{x-t}{\sqrt{t}}\right)$, where $\chi_{0}$ be a smooth function such that

$$
\begin{aligned}
& 0 \leq \chi_{0} \leq 1,\left.\quad \chi_{0}\right|_{(-\infty, b)} \equiv 0,\left.\quad \chi_{0}\right|_{\left(\frac{\tilde{b}+b}{2}, \infty\right)} \equiv 1, \\
& \left|\chi_{0}^{\prime}\right| \leq \frac{A_{0}}{\delta}, \quad\left|\chi_{0}^{\prime \prime}\right| \leq \frac{A_{0}}{\delta^{2}}, \quad \text { where } \delta=(\tilde{b}-b)>0 .
\end{aligned}
$$

Let $\tilde{u}(x, t)=u(x, t) \cdot \chi(x, t)$. Then

$$
\tilde{u}(x, t)=\int_{-\infty}^{\infty} G(x-\xi, t-\alpha t) \tilde{u}(\xi, \alpha t) d \xi+\int_{\alpha t}^{t} d \tau \int_{-\infty}^{\infty} G(x-\xi, t-\tau)\left(\tilde{u}_{\tau}^{\prime}+\Delta \tilde{u}\right)(\xi, \tau) d \xi
$$

where $\alpha t>t_{0}, \alpha \in\left(\frac{1+\sigma_{0}}{1+\sigma}, 1\right)$,

$G(x, t)=\sum_{n=-\infty}^{\infty} G_{n}(t) \delta(n-x), \delta(\cdot)$ the Dirac function,

$G_{n}(t)=\frac{t^{n}}{n !} e^{-t}, \quad n \geq 0, \quad G_{n}(t)=0, \quad n<0 \quad$ the Poisson distribution.

Lemma 2 (corrected lemma 4 from [H1]).

Under assumptions of Propositions 1, 2 and Lemma 1 put $u=f-\varphi^{(-1)}\left(\frac{x}{t}\right)$, where $f$ solution of $(1 \mathrm{~b}),(2)$. Suppose that for some $l \in\{0, \ldots, L-1\}$ we have $\varphi\left(\alpha_{l}^{+}\right)=c_{l}=1$, $\tilde{b}_{l}=\tilde{b}>b_{l}=b \geq O\left(\frac{1}{\gamma}\right)$. Then function $\tilde{u}(\xi, \tau)=u(\xi, \tau) \cdot \chi(\xi, \tau)$ satisfies relation

$$
\begin{aligned}
& \tilde{u}_{\tau}^{\prime}+\Delta \tilde{u}=-\frac{\xi-\tau}{\tau} \Delta u \cdot \chi-\frac{1}{2} \dot{\varphi}\left(\varphi^{(-1)}\left(\frac{\xi}{\tau}\right)\right)\left(\Delta u^{2}\right) \cdot \chi-\frac{u}{\tau} \cdot \chi+ \\
& \Delta u \cdot \Delta \chi+u\left(\chi_{\tau}^{\prime}+\Delta \chi\right)+O\left(\frac{\gamma^{2}}{\tau^{2}}\right), \quad \tau \geq \tau_{0} .
\end{aligned}
$$


Lemma 3 (corrected lemma 5 from [H1]).

Under assumptions of Lemmas 1,2 we have the following representation formula for $\Delta u(x, t)$, if

$$
\begin{gathered}
(x, t) \in \Omega=\left\{(x, t): t^{*}=\alpha t>t_{0}, x \geq t+\frac{1}{2}(b+\tilde{b}) \sqrt{t}\right\}, \\
\Delta u=\sum_{k=1}^{5} I_{k} u, \text { where } \\
I_{0} u=-\int_{\alpha t}^{t} d \tau \int_{\bar{\xi} \geq b} \Delta_{x} G(x-\xi, t-\tau) \frac{\xi-\tau}{\tau} \Delta_{\xi} u(\xi, \tau) \chi(\xi, \tau) d \xi \\
I_{1} u=-\int_{\alpha t}^{t} d \tau \int_{\bar{\xi} \geq b} \Delta_{x} G(x-\xi, t-\tau) \cdot \frac{1}{2} \dot{\varphi}\left(\varphi^{(-1)}\left(\frac{\xi}{\tau}\right)\right) \Delta_{\xi} u^{2}(\xi, \tau) \cdot \chi(\xi, \tau) d \xi, \\
I_{2} u=\int_{\bar{\xi} \geq b}^{t} \Delta_{x} G\left(x-\xi, t-t^{*}\right) u\left(\xi, t^{*}\right) \chi\left(\xi, t^{*}\right) d \xi \\
I_{3} u=\int_{\alpha t}^{t} d \tau \int_{\bar{\xi} \geq b} \Delta_{x} G(x-\xi, t-\tau)\left(u \chi_{\tau}^{\prime}+u \cdot \Delta \chi\right)(\xi, \tau) d \xi, \\
I_{5} u=-\int_{\alpha t}^{t} d \tau \int_{\bar{\xi} \geq b} \Delta_{x} G(x-\xi, t-\tau)\left[\Delta_{\xi} u(\xi, \tau) \cdot \Delta_{\xi} \chi(\xi, \tau)+O\left(\frac{1}{\tau^{2}}\right)\right] d \xi \\
I_{4} u(x-\xi, t-\tau) \frac{u(\xi, \tau)}{\tau} \chi(\xi, \tau) d \xi \\
\int_{\bar{\xi} \geq b}
\end{gathered}
$$

where $\bar{\xi}=\frac{t-\tau}{\sqrt{\tau}}$.

\section{Proof.}

Putting formula (30) into (29) and applying $\Delta_{x}$ to the left- and right-hand sides of (29), we obtain (31).

Lemma 4 ([HST] lemma 6(iv) $+[\mathrm{H} 1]$ lemma 6).

Under assumptions of Lemmas 2,3 we have

$$
\begin{aligned}
& \int_{\xi}\left|\Delta_{\xi} G(x-\xi, t-\tau)\right| d \xi=\min \left\{2, O\left(\frac{1}{\sqrt{t-\tau}}\right)\right\}, \\
& \int_{\alpha t}^{t} d \tau \int_{\xi}\left|\Delta_{\xi} G(x-\xi, t-\tau)\right| d \xi=\sqrt{1-\alpha} O(\sqrt{t})+O(1) .
\end{aligned}
$$




\section{Proof.}

Statement (32) is exactly Lemma 6(iv) from [HST]. Statement (33) follows from (32) by the following way

$$
\begin{aligned}
& \int_{\alpha t}^{t} d \tau \int_{\xi}\left|\Delta_{\xi} G(x-\xi, t-\tau)\right| d \xi= \\
& \int_{\alpha t}^{t-1} d \tau \int_{\xi}\left|\Delta_{\xi} G(x-\xi, t-\tau)\right| d \xi+\int_{t-1}^{t} d \tau\left|\Delta_{\xi} G(x-\xi, t-\tau)\right| d \xi \leq \\
& \int_{\alpha t}^{t-1} d \tau O\left(\frac{1}{\sqrt{t-\tau}}\right)+2 \int_{t-1}^{t} d \tau \leq 2-\left.O(\sqrt{t-\tau})\right|_{\alpha=t} ^{t-1}=O(1)+\sqrt{(1-\alpha)} O(\sqrt{t}) .
\end{aligned}
$$

\section{Lemma 5.}

Let under assumptions of Lemmas 2,3 we have $\frac{1}{\gamma} \leq b_{1}<\tilde{b}_{1}<\tilde{b}_{2}<b_{2}, \chi=\chi_{0}\left(\frac{x-t}{\sqrt{t}}\right)$, $\chi_{0}=1$, on $\left[\tilde{b}_{1}, \tilde{b}_{2}\right], \operatorname{supp} \chi_{0} \subset\left[b_{1}, b_{2}\right], \delta=\min \left\{\tilde{b}_{1}-b_{1}, b_{2}-\tilde{b}_{2}\right\}, \Delta_{x} \chi=O\left(\frac{1}{\delta \sqrt{t}}\right), \alpha<1$. Then

$$
\begin{aligned}
&\left|J_{2} u\right|=O\left(\frac{\gamma}{\sqrt{1-\alpha} \dot{\varphi}\left(\varphi^{(-1)}\left(\frac{x}{t}\right)\right) \cdot(t+1)}\right) \\
&\left|J_{3} u\right|=O\left(\frac{\gamma \tilde{b}_{2} \sqrt{1-\alpha}}{\dot{\varphi}\left(\varphi^{(-1)}\left(\frac{x}{t}\right)\right) \cdot(t+1)}\right)\left(\frac{1}{\delta^{2}}+\frac{\tilde{b}_{2}}{\delta}\right) \\
&\left|J_{4} u\right|=\sqrt{1-\alpha} O\left(\sup \frac{|u(\xi, \tau)|}{\tau}\right) \cdot \sqrt{t+1}=O\left(\frac{\gamma}{t+1}\right) \sqrt{1-\alpha}, \quad \text { where } \\
& \bar{\xi} \geq b_{1}, \quad \alpha t<\tau<t, \quad t \geq t_{0}, \\
&\left|J_{5} u\right|=\sqrt{1-\alpha} O\left(\frac{1}{\delta}\right)\|\Delta u\|+O\left(\frac{1}{(t+1)^{3 / 2}}\right), \quad t \geq t_{0}, \\
& \quad\left|J_{0} u\right| \leq \sqrt{1-\alpha} \sup _{\bar{\xi} \geq b}\left|\frac{\xi-\tau}{\tau}\right| \cdot O(\sqrt{t}) \chi(\xi, \tau) \cdot|\Delta u(\xi, \tau)| \leq \\
& \quad \sqrt{1-\alpha} b_{2} O\left(\frac{\sqrt{t}}{\sqrt{\alpha t}}\right)\|\Delta u\| \leq \frac{\sqrt{1-\alpha}}{\alpha} b_{2}\|\Delta u\|, \\
&\left|J_{1} u\right| \leq \sqrt{1-\alpha} \sup _{\xi \in\left[b_{1}, b_{2}\right]}\left|\dot{\varphi}\left(\varphi^{(-1)}\left(\frac{\xi}{\tau}\right)\right)\right| \cdot\left|\Delta_{\xi} u^{2}(\xi, \tau)\right| \cdot O(\sqrt{t}) \leq \\
& \sqrt{1-\alpha} O\left(\frac{\gamma}{\sqrt{\alpha t}} \cdot \sqrt{t}\right)\|\Delta u\| .
\end{aligned}
$$

\section{Proof.}

Estimate of $J_{2} u$ follows from Lemma 7 of [HST]. Estimate of $J_{3} u$ follows from Lemma 7 of [HST] and Lemma 4. Estimate of $J_{4} u$ follows from Lemma 2 and Lemma 4. Estimate of $J_{5} u$ follows from Lemma 4 and estimate $\left|\Delta_{\xi} \chi(\xi, \tau)\right|=O\left(\frac{1}{\sqrt{\tau} \delta}\right)$. Estimate of $J_{0} u$ follows 
from Lemma 4 and inequality $|\bar{\xi}|=\left|\frac{\xi-\tau}{\sqrt{\tau}}\right| \leq b_{2}$. Estimate of $J_{1} u$ follows from Lemma 2 and Lemma 4.

Lemma 6 (corrected and improved Lemma 8 in [H1]).

Under assumptions of Lemmas 2,3 we have

$$
|\Delta u|=O\left(\frac{\gamma}{\dot{\varphi}\left(\varphi^{(-1)}\left(\frac{x}{t}\right)\right) \cdot(t+1)}\right),
$$

where $x \in[t+b \sqrt{t}, t+\tilde{b} \sqrt{t}], t \geq t_{0}$.

\section{Proof.}

From (31) we obtain

$$
\Delta u-I_{0} u-I_{1} u-I_{5} u=I_{2} u+I_{3} u+I_{4} u .
$$

From Lemma 5 we obtain equality

$$
\begin{aligned}
& \Delta u-I_{0} u-I_{1} u-I_{5} u=\Delta u\left[1-\sqrt{1-\alpha}\left(b_{2}+\gamma+O\left(\frac{1}{\delta}\right)\right]+O\left(\frac{1}{(t+1)^{3 / 2}}\right)=\right. \\
& I_{2} u+I_{3} u+I_{4} u=O\left(\frac{\gamma}{t+1}\right)\left[\frac{1}{\dot{\varphi}\left(\varphi^{(-1)}\left(\frac{x}{t}\right)\right) \cdot \sqrt{1-\alpha}}+\frac{\sqrt{1-\alpha} \tilde{b}_{2}}{\dot{\varphi}\left(\varphi^{(-1)}\left(\frac{x}{t}\right)\right)} \times\right. \\
& \left.\left(\frac{1}{\delta^{2}}+\frac{\tilde{b}_{2}}{\delta}+\sqrt{1-\alpha}\right)\right] .
\end{aligned}
$$

If $\sqrt{1-\alpha}\left(b_{2}+\gamma+O\left(\frac{1}{\delta}\right)\right)<1$ and $\gamma$ is small enough then

$$
|\Delta u| \leq \operatorname{const}\left(b_{2}, \delta\right) O\left(\frac{\gamma}{t+1}\right)\left(\frac{1}{\dot{\varphi}\left(\varphi^{(-1)}\left(\frac{x}{t}\right)\right)}\right) .
$$

\section{Proof of Proposition 2.}

Let $c_{l}=\varphi\left(\alpha_{l}^{+}\right)=1, x=c_{l} t+b_{l}^{*} \sqrt{t}$, where $b_{l}^{*} \in\left[b_{l}, \tilde{b}_{l}\right]$.

Formula $f=\varphi^{(-1)}\left(\frac{x}{t}\right)+u$ and Lemma 6 imply

$$
\begin{aligned}
& \Delta f=\Delta \varphi^{(-1)}\left(\frac{x}{t}\right)+\Delta u=\frac{1}{\dot{\varphi}\left(\alpha_{l}^{+}+O\left(\frac{1}{\sqrt{t}}\right)\right)(t+1)}+\Delta u= \\
& \frac{1}{\varphi^{\prime}\left(\alpha_{l}^{+}\right)(t+1)}+O\left(\frac{\gamma}{\varphi^{\prime}\left(\alpha_{l}^{+}\right)(t+1)}\right), \quad \text { if } t \geq t_{0} .
\end{aligned}
$$

Proposition 2 is proved.

5. Multidimensional Burgers type equations. Systems of conservation laws. Conjectures.

Multidimensional analogues of difference-differential Burgers type equations were proposed in Henkin, Polterovich, 1991 as evolutionary equations for efficiency distributions under several efficiency indicators. 
Let $m, n$ are levels of two efficiency parameters, $f_{m, n}$ be the proportion of firms which are at the level $(m, n)$.

Let $F_{m, n}=\sum_{k=1}^{m} \sum_{r=1}^{n} f_{k, r}$ be the corresponding distribution function.

Hypotheses: The transition from state $(m, n)$ can be into one of two higher levels $(m+1, n)$ and $(m, n+1)$. The proportion of firms per unit of time moving from the state $(m, n)$ to the state $(m+1, n)$ is proportional to the share of firms being in the state $(m, n)$ and the share of firms being in the state $(m, n)$ and the proportion coefficient is positive function of the share of more advanced firms according to the first indicator. A similar hypothesis is admitted for the transition from $(m, n)$ to $(m, n+1)$.

These assumptions lead to the following equation:

$$
\frac{d F_{m, n}}{d t}=\varphi_{1}\left(F_{m}^{(1)}\right)\left(F_{m-1, n}-F_{m, n}\right)+\varphi_{2}\left(F_{n}^{(2)}\right)\left(F_{m, n-1}-F_{m, n}\right)
$$

where

$$
\begin{aligned}
& F_{m}^{(1)}=\sup _{n} F_{m, n}, \quad F_{n}^{(2)}=\sup _{m} F_{m, n} \\
& F_{0, n}(t) \equiv 0, \quad F_{m, 0}(t) \equiv 0 \\
& F_{m, n}(0)=1, \quad m \geq m_{0}, \quad n \geq n_{0}
\end{aligned}
$$

Equation (34) with boundary conditions (35) implies equations

$$
\begin{aligned}
\frac{d F_{m}^{(1)}}{d t} & =\varphi_{1}\left(F_{m}^{(1)}\right)\left(F_{m-1}^{(1)}-F_{m}^{(1)}\right) \\
\frac{d F_{n}^{(2)}}{d t} & =\varphi_{2}\left(F_{n}^{(2)}\right)\left(F_{n-1}^{(2)}-F_{n}^{(2)}\right) .
\end{aligned}
$$

Let functions $\varphi_{1}, \varphi_{2}$ satisfy assumptions $1-3$ of $\S 2$. Theorem 2 , results of [BP], [Con] and $[\mathrm{HP} 1]$ motivate the following conjecture.

Conjecture.

If $\left\{F_{m, n}(t)\right\}$ satisfy $(34),(35)$, then

$$
\sup _{m, n}\left|F_{m, n}(t)-F_{m}^{(1)}(t) F_{n}^{(2)}(t)\right| \rightarrow 0, \quad t \rightarrow \infty .
$$

This statement was proved in [HP1] under condition $L=0$ in assumptions of $\S 2$ for $\varphi_{1}, \varphi_{2}$.

\section{Multidimensional analogues of Burgers differential equations}

(Viscous conservation laws in dimension $n \geq 1$ ).

Let $F(x, t), x \in \mathbb{R}^{n}, t \in \mathbb{R}_{+}^{1}$, be solution of viscous conservation law in dimension $n \geq 1$ :

$$
\frac{\partial F}{\partial t}+\sum_{j=1}^{n} \varphi_{j}(F) \frac{\partial F}{\partial x_{j}}=\varepsilon \Delta F, \varepsilon>0
$$


where functions $\left\{\varphi_{j}\right\}$ satisfy assumptions 1-3 of $\S 2$.

Let

$$
\begin{aligned}
& F(x, 0)=f\left(x_{1}\right)+f^{0}(x), \text { where } \\
& f\left(x_{1}\right)=\left\{\begin{array}{cc}
\alpha^{ \pm}, & \text {if } \pm x_{1}> \pm x_{1}^{ \pm}, \\
f^{0}(x), & \text { if } x_{1} \in\left[x_{1}^{-}, x_{1}^{+}\right], x_{1} \in \mathbb{R},
\end{array}\right.
\end{aligned}
$$

and $f^{0}(x)$ be bounded function with compact support in $\mathbb{R}^{n}$. Theorem 2 and results of Bauman, Phillips, 1986, Weinberger, 1990, Goodman, Miller, 1999, and Hoff, Zumbrun, 2000, motivate the following conjecture.

\section{Conjecture.}

If function $F(x, t), x \in \mathbb{R}^{n}, t \in \mathbb{R}_{+}^{1}$, satisfy (36), (37), then

$$
\left(F(x, t)-\hat{f}_{1}\left(x_{1}, x^{\prime}, t\right)\right) \Longrightarrow 0, t \rightarrow \infty \text {, where } x=\left(x_{1}, \ldots, x_{n}\right)=\left(x_{1}, x^{\prime}\right) \in \mathbb{R}^{n} \text {, }
$$

$$
\begin{aligned}
& \hat{f}_{1}\left(x_{1}, x^{\prime}, t\right)= \\
& \left\{\begin{array}{cl}
\tilde{f}_{l, 1}\left(x_{1}-c_{l} t-o\left(x^{\prime}, t\right)\right), & \text { if }\left|x_{1}-c_{l} t\right|<A \sqrt{t}, l=0, \ldots, L, \\
\varphi_{1}^{(-1)}\left(\frac{x_{1}}{t}\right), & \text { if } c_{l}(t)+A \sqrt{t} \leq x_{1} \leq c_{l+1}(t)-A \sqrt{t}, l=0, \ldots, L-1, \\
\alpha^{-}, & \text {if } x_{1} \leq c_{0} t-A \sqrt{t}, \\
\alpha^{+}, & \text {if } x_{1} \geq c_{L} t+A \sqrt{t},
\end{array}\right.
\end{aligned}
$$

$\left\{c_{l}\right\}$ satisfy (5a), (6a) with function $\varphi=\varphi_{1},\left\{\tilde{f}_{l, 1}\right\}$ - travelling wave solutions of (1a) with $\varphi=\varphi_{1}$ and $\varepsilon=1$, as in Proposition 1 ,

$$
\sup _{x^{\prime}}\left|\frac{o\left(x^{\prime}, t\right)}{t}\right| \rightarrow 0, \quad t \rightarrow \infty
$$

The fundamental applications of multidimensional Burgers type equations for study of the large-scale structure of the Universe were discovered by Zeldovich, 1970, and Gurbatov, Saichev, Shandarin, 1989, through the analysis of the system

$$
\begin{aligned}
& \frac{\partial \vec{v}}{\partial t}+\vec{v} \cdot \nabla \vec{v}=\varepsilon \Delta \vec{v}, \quad \frac{\partial \rho}{\partial t}+\nabla(\rho \vec{v})=0, \\
& \vec{v}(x, 0)=\vec{v}_{0}(x), \quad \rho(x, 0)=\rho_{0}(x)
\end{aligned}
$$

where $x \in \Omega \subset \mathbb{R}^{3}, \vec{v}(x, t)$ - is the velocity field, $\rho(x, t)$ - is the mass density.

Under conditions that $\varepsilon=+0$, the initial distribution of mass $\rho_{0}$ is uniform in convex $\Omega \subset \mathbb{R}^{3}$ and the actual distribution of mass in $\Omega$ is known, this model, combined with technique of Monge-Ampere-Kantorovich optimal mass transportation, was recently applied to the reconstruction of initial velocity $\vec{v}_{0}(x)$ and, as consequence, to reconstruction of $\vec{v}(x, t) \forall x \in \Omega, t \geq 0$ (see [FMMS]).

\section{Systems of Burgers type equations (systems of conservation laws)}

The problems of finding the asymptotics $(t \rightarrow \infty)$ of solutions for systems of Burgers type in one spatial variable have been deeply studied starting from fundamental work of 
Riemann, 1860, for system of barotropic gaz dynamics

$$
\begin{aligned}
& \frac{\partial \rho}{\partial t}+\frac{\partial(\rho v)}{\partial x}=\nu \frac{\partial^{2} \rho}{\partial x^{2}} \\
& \frac{\partial v}{\partial t}+v \frac{\partial v}{\partial x}+\frac{1}{\rho} \frac{\partial P(\rho)}{\partial x}=\varepsilon \frac{\partial^{2} v}{\partial x^{2}}
\end{aligned}
$$

where mass density $\rho \geq 0$, pressure $P(\rho)$ depends only on $\rho, P^{\prime}(\rho) \geq 0$, viscosity coefficient $\varepsilon=+0$, diffusion coefficient $\nu=+0$.

In this direction many important results on existence and asymptotic stability of (viscous) shock profiles have been obtained by Lax, 1957, Gelfand, 1959, Glimm, 1965, T.P.Liu, 1985, Szepessy, Xin, 1993, Howard, Zumbrun, 1998, Yu, 1999, Bianchini, Bressan, $2005 \ldots$

Results of the type Theorems 1,2,3 above for systems of Burgers type have not been obtained yet, even for system barotropic gaz dynamics. Some interesting conjectures in this direction are formulated in Gelfand, 1959, Maslov 1988, Serre 2004.

Similar questions for systems of difference-differential Burgers type equations have been considered only recently (see Benzoni-Gavage, Huot, Rousset, 2003).

This subject is especially important for Schumpeterian models of economical developments of several interacting industries.

\section{References.}

[B] H.Bateman, Some recent researches on the motion of fluids, Monthly Weather Rev. 43 (1915), 163-170

[ Be1] V.Belenky, Diagram of growth of a monotonic function and a problem of their reconstruction by the Diagram, Preprint, Central Economics and Mathematical Institut, Academy of Sciences of the USSR, Moscow, 1990, 1-44 (in Russian)

[ Be2] V.Belenky, Variations on the theme "Modelisation of convergent evolutionary processes", In: "Probabilities models of mathematical economics" , Moscow, CEMI RAN, 1996

[ Be3] V.Belenky, Alternative version of evolution model of Polterovich-Henkin "diffusion of technologies", In: "Analyse and modelisation of economical processes", Moscow, CEMI RAN, 2007, 115-142

[ BHR] S.Benzoni-Gavage, P.Huot, F.Rousset, Nonlinear stability of semidiscrete shock waves, SIAM J.Math.Anal, 2003

[ BiB] S.Bianchini, A.Bressan, Vanishing viscosity solutions of nonlinear hyperbolic systems, Ann. Math., 2005

[ BL] S.E.Buckley, M.C.Leverett, Mechanism of fluid displacement in sands, A.I.M.E. 146 (1942), 107-116

[ BP] P.Bauman, D.Phillips, Large-time behaviour of solutions to a scalar conservation law in several space dimensions, Transl.Amer.Math.Soc. 298 (1986), 401-419

[ Bu1] J.M.Burgers, Mathematical examples illustrating relations occurring in the theory of turbulent fluid motion, Verhand. Kon. Neder. Akad. Wetenschappen, Afd. Natuurkunde, Eerste Sectie 17 (1939), 1-53 
[ Bu2] J.M.Burgers, Application of a model system to illustrate some points of the statistical theory of free turbulence, Proc.Acad.Sci. Amsterdam 43 (1940), 2-12

[ Co] J.D.Cole, On a quasi-linear parabolic equation occurring in aerodynamics, Quarterly of Appl.Math., 9 (1951), 225-236

[ Con] E.D.Conway, The formation and decay of schocks for a conservation law in several dimensions, Arch.Rat. Mech. Anal. 64(1), 1977,47-57

[ ES] S.Engelberg, S.Schochet, Nonintegrable perturbations of scale viscous shock profiles, Asymptotic Analysis 48, 2006, 121-140

[ EO] B.Engquist, S.Osher, One-sided difference approximations for nonlinear conservation laws, Math.Comp. 36(154) (1981), 321-351

[F] V.A.Florin, Some of the simplest nonlinear problems arising in the consolidation of wet soil, Izv.Akad.Nauk SSSR, Otdel. Tekhn. Nauk, 9 (1948), 1389-1397 (in Russian)

[ FS] H.Freistuhler, D.Serre, $L^{1}$-stability of shockwaves in scalar viscous conservation laws, Comm. Pure Appl.Math. 51, 1998, 291-301

[FMMS] U.Frisch, S.Matarrese, R.Mohayaee, A.Sobolevskii, A reconstruction of the initial condition of the Universe by optimal mass transportation, Nature 417, 2002, 260-262

[ G] I.M.Gelfand, Some problems in the theory of quasilinear equations, Usp.Mat.Nauk 14 (1959), 87-158 (in Russian); Amer.Math.Soc. Translations 33 (1963), 295-381

[GM] J.Goodman, J.Miller, Long-time behaviour of scalar viscous shock fronts in two dimensions, J.Dynam.Differential Equations, 11, 1999, 255-277

[ Ga1] A.V.Gasnikov, Time asymptotic behaviour of the solution to a quasilinear parabolic equation, Computational Mathematics and Mathematical Physics 46 (2006), 21362153

[ Ga2] A.V.Gasnikov, Convergence in form of solutions of the Cauchy problem for quasilinear equation of parabolic type with monotone initial condition to system of waves, J.Computer Math. and Math. Physics, 48(8), 2008, 1458-1487

[ Ga3] A.V.Gasnikov, Time asymptotic behaviour of initial Cauchy problem for conservation law with nonlinear divergent viscosity, Izvestia RAN, Mathematical Serie 76(6) (2009), 36-76

[ Ga4] A.V.Gasnikov, Time asymptotic structure of solutions to the Riemann's type initial Cauchy problem for the conservation law, Proceedings of Moscow Institut of Physics and Technology 1(4) (2009), 120-125 (in Russian)

[GLPS] L.M.Gelman, M.I.Levin, V.M.Polterovich, V.A.Spivak, Modelling of dynamics of enterprises. Distribution by efficiency levels for the Ferrous Metallurgy, Economic and mathematical methods 29(3) (1993), 1071-1083

[HKT1] R.Hanel, P.Klimek, S.Thurner, Studies in the physics of evolution: creation, formation, distruction, Proc. of SPIE 6802 (2008) 680206

[HKT2] R.Hanel, P.Klimek, S.Thurner, Schumpeterian economic dynamics as a quantifiable model of evolution, New J.Phys., 12, 2010, 075029

[ HHL] A.Harten, J.M.Hyman, P.D.Lax, On finite-difference approximations and entropy conditions for shocks, Comm.Pure Appl.Math. 29 (1976), 297-322

[ H1] G.Henkin, Asymptotic structure for solutions of the Cauchy problem for Burgers type equations, J.Fixed Point Theory Appl., 1 , 2007, 239-291 
[ H2] G.Henkin, Burgers type equations and Schumpeterian dynamics, Conference "General Equilibrium Analysis", Higher school of Economics, June 2011, Preprint, 11 p.

[ Ho] E.Hopf, The partial differential equation $u_{t}+u u_{x}=\mu u_{x x}$, Comm.Pure Appl.Math. 3 (1950), 201-230

[ HP1] G.M.Henkin, V.M.Polterovich, Schumpeterian dynamics as a nonlinear wave theory, J.Math.Econom. 20 (1991), 551-590

[ HP2] G.M.Henkin, V.M.Polterovich, A difference-differential analogue of the Burgers equation: stability of the two-wave behaviour, J.Nonlinear Sci. 4 (1994), 497-517

[ HP3] G.M.Henkin, V.M.Polterovich, A difference-differential analogue of the Burgers equation and some models of economic development, Discrete Contin.Dynam.Systems 4 (1999), 697-728

[ HS] G.M.Henkin, A.A.Shananin, Asymptotic behaviour of solutions of the Cauchy problem for Burgers type equations, J.Math.Pure Appl. 83 (2004), 1457-1500

[HST] G.M.Henkin, A.A.Shananin, A.E.Tumanov, Estimates for solutions of Burgers type equations and some applications, J.Math.Pures Appl. 84 (2005), 717-752

[ HZ] D.Hoff, K.Zumbrun, Asymptotic behaviour of multidimensional viscous shock fronts, Indiana Univ.Math.J. 49 (2000), 427-474

[HowZ] D.Howard, K.Zumbrun, (1998), Poinwise semigroup methods and stability of viscous shock waves, Indiana Univ. Math. J. 47, 63-185

[ IO] A.M.Iljin, O.A.Oleinik, Asymptotic long-time behaviour of the Cauchy problem for some quasilinear equation, Mat.Sbornik 51 (1960), 191-216 (in Russian)

[ I1] K.Iwai, Schumpeterian dynamics, Part I: An evolutionary model of innovation and imitation, J. of Economic behaviour and organization 5 (1984), 159-190

[ I2] K.Iwai, Schumpeterian dynamics, Part II: Technological progress, firm growth and "economic selection", J. of Economic behaviour and organization 5 (1984), 287-320

[ KPP] A.Kolmogorov, I.Petrovski, N.Piskunov, A study of the diffusion equation with increase of the amount of substance and its application to biological problem, Bull. Moscow Univ. Math.Mech., 1(6), 1937, 1-26

[KP] S.N.Kruzkov, N.S.Petrosjan, Asymptotic behaviour of solutions of Cauchy problem for nonlinear equations of first order, Russian Math. Surveys (Uspehi Mat. Nauk), 42(5), 1987, 3-40

[ Ku] N.Kuznetsov, Accuracy of some approximate methods for computing the weak solutions of a first order quasi-linear equation, J.Comp.Math. and Math. Phys., 16, 1976, 105-119

[ La] P.D.Lax, Hyperbolic systems of conservation laws, II, Comm. Pure Appl. Math. 10 (1957), 537-566

[L1] T.-P.Liu, Admissible solutions of hyperbolic conservation laws, Memoirs of the Amer. Math. Soc., 240, 1981, 1-78

[ L2] T.-P.Liu, Nonlinear stability of shock waves for viscous conservation laws, Memoirs of the Amer. Math. Soc., Providence, R.I., 328, 1985

[LW] M.J.Lighthill, G.B.Whitham, On kinematic waves II: Theory of traffic flow on long crowded roads, Proc. R. Soc. London Ser. A 229 (1955), 281-345

[ LMN] T.-P.Liu, A.Matsumura, K.Nishihara, Behaviours of solutions for the Burgers equation with boundary corresponding to rarefaction waves, SIAM J.Math.Anal. 29 (1998), 


\section{3-308}

[ M] V.Maslov, Asymptotic methods and theory of perturations, M. Nauka, 1988 (in Russian)

[ MV] M.Mejai, Vit.Volpert, Convergence to systems of waves for viscous scalar conservation laws, Asymptotic analysis 20 (1999), 351-366

[ O] O.A.Oleinik, Uniqueness and stability of the generalized solution of the Cauchy problem for a quasi-linear equation, Usp.Mat.Nauk 14 (1959), 167-170 (in Russian); Amer. Math.Soc. Translations 33 (1963), 285-290

[P] N.S.Petrosjan, About asymptotics of solutions of the Cauchy problem for quasilinear equation of first order with nonconvex state function, Russian Math. Surveys (Uspehi Mat. Nauk), 38(2), 1983, 213-214

[ PH1] V.M.Polterovich, G.M.Henkin, An evolutionnary model with interaction between development and adoption of new technologies, Econom. Math.Methods 24 (1988), 1071-1083 (in Russian); Matecon (1988) 24(6), p.3-19 (in English)

[ PH2] V.M.Polterovich, G.M.Henkin, Diffusion of technologies and economic growth, Preprint CEMI Academy of Sciences of the USSR, 1988, 1-44 (in Russian)

[ PH3] V.M.Polterovich, G.M.Henkin, An evolutionary model of economic growth, Econom. Math. Methods 25(3) (1989), 518-531 (in Russian); Matecon (1990) 26(3), 44-64 (in English)

[ R] B.Riemann, Uber die Fortpflanrung ebener Luftwellen von endlicher

Schwingungsweite, Gottingen Abh.Math. cl 8, 1960, 43-65

[ S1] J.A.Schumpeter, Theorie der wirtschaftlichen Entwicklung, Wien, 1911

[S2] J.A.Schumpeter, Business Cycles: A Theoretical, Historical and Statistical Analysis of the Capitalist Process, McGraw-Hill, NY, 1939

[ Se1] D.Serre, Systems of Conservation Laws, I.Cambridge Univ. Press, 1999

[ Se2] D.Serre, $L^{1}$-stability of nonlinear waves in scalar conservation laws, In: C.Dafermos and E.Feireis (eds), Handbook of Differential Equations, 2004, 473-553

[ST] A.A.Shananin, Y.M.Tashlitskaya, Investigation of a model of propagation of new technologies, Preprint 2000, Moscow Computer Centre RAN 1-50 (in Russian)

[ SZ] A.Szepessy, Z.Xin, Nonlinear stability of viscous shock waves, Arch.Rat.Mech.Anal. 122, 1993, 53-103

[ W] H.F.Weinberger, Long-time behaviour for a regularized scalar conservation law in the absence of genuine nonlinearity, Ann.Inst.H.Poincare Anal.Nonlinéaire (1990), 407-425

[ Y] S.H.Yu, Zero-dissipation limit of solutions with shocks for systems of hyperbolic conservation laws, Arch. Ration. Mech. Anal. 146, 1999, 275-370

[ Z] Ya.Zeldovich, Gravitational instability: an approximate theory for large density perturbations, Astron. Astrophys. 5, 1970, 84-89 